\title{
Characteristics of regionally reflectionless potentials
}

\author{
Eric Kincanon
}

\author{
Department of Physics, Gonzaga University, Spokane, WA, United States \\ E-mail: kincanon@gonzaga.edu
}

\begin{abstract}
Copyright $($ ) 2015 Eric Kincanon. This is an open access article distributed under the Creative Commons Attribution License, which permits unrestricted use,
\end{abstract} distribution, and reproduction in any medium, provided the original work is properly cited.

\begin{abstract}
This paper considers potentials that are reflectionless for a range of energies and not reflectionless elsewhere. The focus of this paper is to see if reflection coefficients of this type have characteristics that would make it possible to solve for the associated potentials using known methods. Using the Gelfand-Levitan equation it is shown that the spectral measure function has several limits that match well to known solved cases. Thus, it seems that pursuing cases of potentials of this type is reasonable.
\end{abstract}

Keywords: Gelfand-Levitan; Inverse Scattering; Relfectionless Potentials.

\section{Introduction}

Inverse scattering on the line is a well-known method to determine the potential $\mathrm{V}(\mathrm{r})$ given the reflection coefficient $\mathrm{R}(\mathrm{k})$. This is done by using the Gelfand-Levitan Equation [1-3]:

$K(r, s)+G(r, s)+\int_{-\infty}^{r} K(r, t) G(t, s) d t=0$

Where $\mathrm{G}(\mathrm{r}, \mathrm{s})$, the spectral measure function, is the Fourier transform of the reflection coefficient $\mathrm{R}(\mathrm{k})$ :

$G(r, s)=\frac{1}{2 \pi} \int_{-\infty}^{+\infty} R(k) e^{-i k(r+s)} d k$

And $\mathrm{K}(\mathrm{r}, \mathrm{s})$ is related to the potential by:

$V(r)=-2 \frac{d K(r, r)}{d r}$

The typical procedure is to find $\mathrm{V}(\mathrm{r})$ by taking the Fourier transform of $\mathrm{R}(\mathrm{k})$ to get $\mathrm{G}(\mathrm{r}, \mathrm{s})$ and then solve (1) for $\mathrm{K}(\mathrm{r}, \mathrm{s})$. Then (3) can be used to find the potential. The challenge arises in solving (1). This is typically done by successive iteration [4].

A case of particular interest is that of reflectionless potentials [5-8]. These are cases where the reflection coefficient is zero but the potential is non-zero. This is possible because one can have the case of $\mathrm{R}(\mathrm{k})$ being zero and $\mathrm{G}(\mathrm{r}, \mathrm{s})$ being non-zero if bound states exist [9], [10]. This paper considers the case of a reflection coefficient that is zero in a region and non-zero elsewhere. This would correspond to a potential that did not reflect a wave for a range of energies but did in general reflect outside of that range. The goal of this paper is to see what particular characteristics these potentials have that can aid in calculating corresponding potentials. 


\section{Regionally Reflectionless Potentials}

Consider a reflection coefficient $\mathrm{r}(\mathrm{k})$ :

$\mathrm{r}(\mathrm{k})=\mathrm{R}(\mathrm{k})\left[1-\mathrm{N}\left(\mathrm{k}_{1}\right)\right]$

Where $\mathrm{R}(\mathrm{k})$ is any physically allowable reflection coefficient and $\mathrm{N}\left(\mathrm{k}_{1}\right)=0$ if $|\mathrm{k}| \leq \mathrm{k}_{1}$ and $\mathrm{N}\left(\mathrm{k}_{1}\right)=1$ otherwise. To find the potential associated with this $r(k)$, the spectral measure function $G(r, s)$ must be found. Combining (2) and (4) gives:

$\mathrm{G}(\mathrm{r}, \mathrm{s})=\frac{1}{2 \pi} \int_{-\infty}^{+\infty} \mathrm{R}(\mathrm{k}) \mathrm{e}^{-\mathrm{ik}(\mathrm{r}+\mathrm{s})} \mathrm{dk}-\frac{1}{2 \pi} \int_{-\infty}^{+\infty} \mathrm{R}(\mathrm{k}) \mathrm{N}\left(\mathrm{k}_{1}\right) \mathrm{e}^{-\mathrm{ik}(\mathrm{r}+\mathrm{s})} \mathrm{dk}$

where the right hand side of (4) has been broken into two terms.

The first term of the right hand side is defined as $\mathrm{g}(\mathrm{r}, \mathrm{s})$. The second term is given by:

$\frac{2 \sin \left[\mathrm{k}_{1}(\mathrm{r}+\mathrm{s})\right]}{\mathrm{r}+\mathrm{s}}=\frac{1}{2 \pi} \int_{-\infty}^{+\infty} \mathrm{R}^{*}(\mathrm{k}) \mathrm{e}^{-\mathrm{ik}(\mathrm{r}+\mathrm{s})} \mathrm{dk}$

Combining this with (5), the definition of $\mathrm{g}(\mathrm{r}, \mathrm{s})$, and using the convolution theorem for Fourier transforms, yields:

$G(r, s)=g(r, s)-2 \int_{-\infty}^{+\infty} \frac{\sin \left(k_{1} x\right)}{x} g(r+s-x) d x$

In order to learn more about this G(r,s), a method developed for solving GL can be used [11]. This method solves GL by looking at the expansion of G(r,s) into a sum of separable functions. (This method will be designated SM for Separable Method.) In this case it was assumed that $\mathrm{R}(\mathrm{k})$ in (4) was a physically reasonable function. This means that SM can be used to analyze $\mathrm{g}(\mathrm{r}, \mathrm{s})$, since it is the spectral measure function associated with $\mathrm{R}(\mathrm{k})$. This means that $\mathrm{g}(\mathrm{r}, \mathrm{s})$ can be represented by:

$g(r, s)=\sum_{n=1}^{\infty} a_{n} e^{n(r+s)}$

Putting this into the integrand of (7) yields:

$G(r, s)=g(r, s)-2 \sum_{n=1}^{\infty} a_{n} e^{n(r+s)} \int_{-\infty}^{+\infty} \frac{\sin \left(k_{1} x\right)}{x} e^{n x} d x$

which gives:

$G(r, s)=g(r, s)-2 \sum_{n=1}^{\infty} a_{n} e^{n(r+s)} \tan ^{-1} \frac{k_{1}}{n}$

Again applying (8) yields:

$\mathrm{G}(\mathrm{r}, \mathrm{s})=\sum_{\mathrm{n}=1}^{\infty} \mathrm{a}_{\mathrm{n}} \mathrm{e}^{\mathrm{n}(\mathrm{r}+\mathrm{s})}-2 \sum_{\mathrm{n}=1}^{\infty} \mathrm{a}_{\mathrm{n}} \mathrm{e}^{\mathrm{n}(\mathrm{r}+\mathrm{s})} \tan ^{-1} \frac{\mathrm{k}_{1}}{\mathrm{n}}$

Or:

$\mathrm{G}(\mathrm{r}, \mathrm{s})=\sum_{\mathrm{n}=1}^{\infty} \mathrm{a}_{\mathrm{n}} \mathrm{e}^{\mathrm{n}(\mathrm{r}+\mathrm{s})}\left[1-\tan ^{-1} \frac{\mathrm{k}_{1}}{\mathrm{n}}\right]$

The coefficients in the sum of (8) are detailed in [11]. Using this one could solve for $\mathrm{K}(\mathrm{r}, \mathrm{s})$ in principle using GL. However this is often quite complicated. There are, however some interesting results to note about (12).

\section{Results}

Considering (12) there are conditions worth noting. If (12) is to be used with SM to find $\mathrm{K}(\mathrm{r}, \mathrm{s})$ then it is necessary that the $\mathrm{G}(\mathrm{r}, \mathrm{s})$ in $(12)$ meets the requirements necessary as detailed in [11].

First consider the fact that: 
$\lim _{n \rightarrow \infty} \tan ^{-1} \frac{k_{1}}{n}=0$

This leads to the large $\mathrm{n}$ terms in (12) approaching the corresponding terms in (8). In practice, this simplifies SM. This gives some confidence in solving GL for regionally reflectionless potentials (RRP). RRP behave like acceptable potentials in GL for large values of $\mathrm{n}$ in (12), which means SM should work well.

Another condition worth considering is what happens if the reflectionless region becomes very large. This would be looking at the limit:

$\lim _{\mathrm{k}_{1} \rightarrow \infty}\left(1-2 \tan ^{-1} \frac{\mathrm{k}_{1}}{\mathrm{n}}\right)=1-\pi$

Since this limit is independent of $n,(12)$ is just a constant times (8) for this limit. What this means is that the RRP behaves like (8) for large energies. This again gives support for using SM.

Finally, it is interesting to look at the case of applying (14) and only looking at the first term in the series. This leads to a $\mathrm{G}(\mathrm{r}, \mathrm{s})$ of the form:

$G(r, s)=a_{n}^{\prime} e^{r+s}$

$\mathrm{G}(\mathrm{r}, \mathrm{s})$ of this form have been shown to be associated with the Bargmann potentials [9], [10]. These potentials consist of analytic solutions to GL, again pointing to RRP cases being solvable.

\section{Conclusion}

It has been shown that regionally reflectionless potentials have several characteristics that lead to the possibility of analysis using inverse scattering. In particular, by using the representation presented it seems that a current method of solution (SM) may be fruitful. This method is not trivial but this paper does point to the possible way of identifying regionally reflectionless potentials.

\section{References}

[1] I.M. Gelfand, B.M. Levitan, On the determination of a differential equation by its spectral function, Dokl. Akad. Nauk. USSR 77 (1951) 557560.

[2] I.M. Gelfand, B.M. Levitan, On the determination of a differential equation by its spectral measure function, Izv. Akad. Nauk. SSR 15 (1951) 309-360.

[3] K. Chadan, P.C. Sabatier, Inverse Problems in Quantum Scattering Theory, Springer-Verlag, New York, 1977. http://dx.doi.org/10.1007/9783-662-12125-2.

[4] R. Jost, W. Kohn, On the relation between phase shift energy levels and the potential, Danske Vid. Selsk. Math. Fys. 27 (1953) 3-19.

[5] E. Kincanon, A method to construct reflectionless potentials, Applied Mathematics and Computation, 165 (2005) 565-569. http://dx.doi.org/10.1016/j.amc.2004.04.075.

[6] J.Lekner,Reflectionless Eigenstates of the sech potential, American Journal of Physics, 75 (2007) 1151-1157. http://dx.doi.org/10.1119/1.2787015.

[7] I. Kay, H.E. Moses, Reflectionless transmission through dielectrics and scattering potentials, Journal of Applied Physics, 27 (1956) 15031508. http://dx.doi.org/10.1063/1.1722296.

[8] N. Kiriushcheva, S. Kuzmin, Scattering of a Gaussian wave packet by a reflectionless potential, American Journal of Physics, 66 (1998) 867872. http://dx.doi.org/10.1119/1.18985.

[9] V. Bargmann, Remarks on the determination of the central field of force from the elastic scattering phase shift, Physical Review, 75 (1949) 301-303. http://dx.doi.org/10.1103/PhysRev.75.301.

[10] V. Bargmann, On connections between phase shifts and scattering potential, Review of Modern Physics, 21 (1949) $488-493$. http://dx.doi.org/10.1103/RevModPhys.21.488.

[11] E. Kincanon, Approximate solution it the Gelfand-Levitan equation, Applied Mathematics and Computation, 53 (1993) 121-128. http://dx.doi.org/10.1016/0096-3003(93)90097-X. 